\title{
ON THE EXISTENCE OF UNIFORMLY DISTRIBUTED SEQUENCES IN COMPACT TOPOLOGICAL SPACES. I
}

BY

\author{
V. LOSERT
}

\begin{abstract}
We prove the existence of uniformly distributed sequences for an arbitrary probability measure on a separable dyadic space, e.g. on a separable compact topological group. Some counterexamples for the nonexistence of u.d. sequences in certain dense subsets are given.
\end{abstract}

Let $X$ be a compact topological space, $\mu$ a probability measure on $X$. A sequence $\left(x_{n}\right)$ in $X$ is called $\mu$-uniformly distributed ( $\mu$-u.d.), if

$$
\lim _{N \rightarrow \infty} \sum_{n=1}^{N} f\left(x_{n}\right)=\int f d \mu
$$

holds for any continuous, complex valued function $f$ on $X$ (see [7, p. $171 \mathrm{ff}$.] for basic properties and examples of u.d. sequences).

It follows immediately from the definition that the closure of a u.d. sequence contains the support of the measure. One can therefore restrict oneself to the case of separable spaces. It can be shown that for metrizable $X$ u.d. sequences always exist and behave in much the same way as in the classical case of the torus group. For compact separable topological groups and Haar measure it follows from results in [12] (see also [1], [11]) that u.d. sequences exist. On the other hand it was shown in [9] that on the Stone-Čech compactification of the integers u.d. sequences exist only if the measure is concentrated on a countable set. One of the aims of this paper is to give further conditons for the existence of u.d. sequences.

First we treat the following 'lifting problem': if $\pi: X \rightarrow Y$ is surjective and continuous, $X$ metrizable and if a sequence $\left(y_{n}\right)$ is u.d. with respect to the image measure $\pi(\mu)$ on $Y$, does there always exist a $\mu$-u.d. sequence $\left(x_{n}\right)$ on $X$, for which $\pi\left(x_{n}\right)=y_{n}$ ? The special case where $X=Y \times Z, \pi(y, z)=y$, $\mu=\nu \otimes \rho$ has been considered in [4]. In the general case it turns out that a lifting is possible for any $\pi(\mu)$-u.d. sequence in $Y$, iff the map $\pi$ is open in $\mu$-almost all points of $X$, in particular if $\pi$ is an open map.

Using this result, we can show that for dyadic spaces with an open basis of

Received by the editors February 7, 1978.

AMS (MOS) subject classifications (1970). Primary 10K99; Secondary 22C05, 46E15.

Key words and phrases. Uniform distribution, dyadic spaces, compact topological groups. 
cardinality $\aleph_{1}$ u.d. sequences exist for any measure $\mu$ (a topological space $X$ is called dyadic, if it is a continuous image of $\{0,1\}^{I}$ for some index set $I$, see [2], [3]). In particular, if one assumes the continuum hypothesis, u.d. sequences exist for arbitrary measures on compact separable groups. Finally we give an example which shows that the dyadic spaces form a proper subclass of those spaces for which u.d. sequences exist for any measure.

In the last section we give three counterexamples which show that some properties of u.d. sequences, valid on metrizable spaces, do not carry over to arbitrary separable spaces: it is in general not possible to choose the u.d. sequence in the support of the measure $\mu$. On a topological group $G$ the u.d. sequence cannot be chosen in an arbitrary dense subgroup of $G$. If $\mu$ is the Haar measure on $G$, it has been shown in [11] that it is possible to construct a u.d. sequence in a given countable dense subgroup, but we show that it is not possible to choose it in an arbitrary countable dense subset. (On metrizable groups any dense sequence can be rearranged to a u.d. sequence, compare [7, p. 185], and [6].)

Proposition 1. Let $X, Y$ be compact, metrizable topological spaces, $\pi$ : $X \times Y \rightarrow X$ the projection onto the first coordinate, $\mu$ a probability measure on $X \times Y, \nu=\pi(\mu)$. If $\left(x_{n}\right)$ is $\mu$-u.d. in $X$, there exists a sequence $\left(y_{n}\right)$ in $Y$, such that $\left(x_{n}, y_{n}\right)$ is $\mu$-u.d. in $X \times Y$.

Proof. Let $P_{n}$ be a sequence of partitions of $X$ with the following properties:

(i) $P_{n+1}$ refines $P_{n}$;

(ii) each $A \in P_{n}$ satisfies $\nu(\partial A)=0$ and its diameter is less than $n^{-1}$. ( $\partial A$ denotes the boundary of $A$.)

In a similar fashion we can choose a sequence of partitions $Q_{n}$ of $Y$. By [7, pp. 172, 174] a sequence $\left(x_{n}, y_{n}\right)$ is $\mu$-u.d. iff

$$
\lim N^{-1} \sum_{n=1}^{N} c_{A}\left(x_{n}\right) c_{B}\left(y_{n}\right)=\mu(A \times B)
$$

for $A \in \cup P_{n}, B \in \cup Q_{n}$.

Put $m_{n}=\left|P_{n}\right|\left|Q_{n}\right|\left(\left|P_{n}\right|\right.$ denotes the number of elements of $\left.P_{n}\right)$. We may assume that $m_{n}$ tends to infinity. Assume that $A \in P_{n}$ and that $M$ and $N$ are natural numbers with $N>M$. Then

$$
\begin{aligned}
& \left|(N-M)^{-1} \sum_{n=M+1}^{N} c_{A}\left(x_{n}\right)-\nu(A)\right| \\
& \quad<2 N(N-M)^{-1} \sup _{k>M}\left|K^{-1} \sum_{n=1}^{K} c_{A}\left(x_{n}\right)-\nu(A)\right|
\end{aligned}
$$

and this expression becomes arbitrarily small for large $M$, provided that the 
quotient $(N-M) / N$ does not decrease too fast. Using this observation, one can construct a monotone increasing sequence of natural numbers $N_{k}\left(N_{1}=\right.$ 0 ), and a nondecreasing map $\varphi: \mathbf{N} \rightarrow \mathbf{N}$ with the following properties:

(i) $\left|\left(N_{k+1}-N_{k}\right)^{-1} \sum_{n=1}^{N_{k+1}+1} c_{A}\left(x_{n}\right)-\nu(A)\right|<m_{\varphi(k)}^{-2}$ holds for all $A \in P_{\varphi(k)}$.

(ii) $N_{k+1}-N_{k}>m_{\Phi(k)}^{2}$.

(iii) $\lim _{k \rightarrow \infty} N_{k+1} / N_{k}=1$.

For $A \in P_{\varphi(k)}$ put $I_{A}=\left\{n: N_{k}<n<N_{k+1}, x_{n} \in A\right\}$. For each $B \in Q_{\varphi(k)}$ we can find a natural number $\left|I_{A B}\right|$ such that ||$I_{A B}\left|-\mu(A \times B) \nu(A)^{-1}\right| I_{A}||$ $<1$ and $\Sigma_{B \in Q_{p(k)}}\left|I_{A B}\right|=\left|I_{A}\right|$.

Now choose $y_{n} \in Y$ such that $\left|I_{A B}\right|=\mid\left\{n: N_{k}<n<N_{k+1},\left(x_{n}, y_{n}\right) \in A \times\right.$ $B$ \}. It follows that

$$
\begin{aligned}
\mid\left(N_{k+1}\right. & \left.-N_{k}\right)^{-1} \sum_{n=N_{k}+1}^{N_{k+1}} c_{A \times B}\left(x_{n}, y_{n}\right)-\mu(A \times B) \mid \\
& =\left|\left(N_{k+1}-N_{k}\right)^{-1}\right| I_{A B}|-\mu(A \times B)| \\
& <\left(N_{k+1}-N_{k}\right)^{-1}+\mu(A \times B)\left|\left(N_{k+1}-N_{k}\right)^{-1} \nu(A)^{-1}\right| I_{A}|-1| \\
& <m_{\varphi(k)}^{-2}+\mu(A \times B) \nu(A)^{-1} m_{\varphi(k)}^{-2} \\
& <2 m_{\varphi(k)}^{-2} .
\end{aligned}
$$

Since for $l>k, P_{\varphi(l)}$ (resp. $\left.Q_{\varphi(l)}\right)$ is a refinement of $P_{\varphi(k)}$ (resp. $\left.Q_{\varphi(k)}\right)$ we get:

$$
\left|\left(N_{l+1}-N_{l}\right)^{-1} \sum_{n=N_{l}}^{N_{l+1}} c_{A \times B}\left(x_{n}, y_{n}\right)-\mu(A \times B)\right|<2 m_{\varphi}^{-1}(l) \text {. }
$$

Since $N_{m+1} / N_{m}$ tends to zero we get for $N>N_{0}(l)$ :

$$
\left|N^{-1} \sum_{n=1}^{N} c_{A \times B}\left(x_{n}, y_{n}\right)-\mu(A \times B)\right|<3 m_{\varphi}^{-1}(l) \text {. }
$$

Remarks. (1) An example in [10] shows that Proposition 1 is not true in general for nonmetrizable, compact $X$, even if $\mu=\nu \otimes \rho$ for some measure $\rho$.

(2) If $\mu=\nu \otimes \rho, X, Y$ metrizable, it has been shown in [4] that $\left(x_{n}, y_{n}\right)$ is $\mu$-u.d. in $X$ for almost all sequences in $Y$ (with respect to the product measure $\rho^{N}$ on $\left.Y^{N}\right)$. If $\mu$ is not of the form $\nu \otimes \rho$, it follows that the set of sequences $\left(y_{n}\right)$ for which $\left(x_{n}, y_{n}\right)$ is $\mu$-u.d. has measure zero in $Y^{N}$ with respect to any probability measure $\rho$ on $Y$. For example if $X=Y$ and $\mu$ is the diagonal measure of $\nu$, i.e.

$$
\int_{X \times X} f(x, y) d \mu(x, y)=\int_{X} f(x, x) d \nu(x),
$$

then $\left(x_{n}, y_{n}\right)$ is $\mu$-u.d. iff for any $\varepsilon>0$ the set $\left\{n: d\left(x_{n}, y_{n}\right)>\varepsilon\right\}$ has density zero in $\mathbf{N}$. 
Proposition 2. Let $X, Y$ be compact, metrizable spaces, $\pi: X \rightarrow Y$ continuous, surjective, $d$ a metric for the topology of $Y, \mu$ a probability measure on $X, \nu=\pi(\mu)$. If $\left(y_{n}\right)$ is a $\nu$-u.d. sequence in $Y$, there exists a $\mu$-u.d. sequence $\left(x_{n}\right)$ in $X$, such that $d\left(\pi\left(x_{n}\right), y_{n}\right)$ converges to zero $(n \rightarrow \infty)$.

Proof. We consider the space $C(X)$ of continuous, real valued functions on $X$, equipped with supremum norm. If $X$ is metrizable, this space is separable. Let $\left(f_{n}\right)_{n=1}^{\infty}$ be a countable dense subset in the unit ball of $C(X)$. Put $Z=Y \times[-1,1]^{\mathrm{N}} . \pi_{1}: X \rightarrow Z$ is defined by

$$
\pi_{1}(x)=\left(\pi(x), f_{1}(x), f_{2}(x), \ldots\right) .
$$

Since $\left(f_{n}\right)$ is dense in the unit ball of $C(X), \pi_{1}$ is injective and consequently a homeomorphism onto $\pi_{1}(X)$. Let $p$ be the projection of $Z$ onto $Y, \mu_{1}=\pi_{1}(\mu)$. Since $p \circ \pi_{1}=\pi$, we have clearly $p\left(\mu_{1}\right)=\nu$.

By Proposition 1 we can lift our sequence $\left(y_{n}\right)$ to a $\mu_{1}$-u.d. sequence $\left(z_{n}\right)$ in $Z$. Since the support of the measure $\mu_{1}$ is contained in $\pi_{1}(X)$ and $Z$ is metrizable, we can find a sequence $\left(x_{n}\right)$ in $X$ such that $d\left(\pi_{1}\left(x_{n}\right), z_{n}\right)$ tends to zero ( $d$ denotes again a metric for the topology of $Z$ ). It follows easily that $\pi_{1}\left(x_{n}\right)$ (and therefore $x_{n}$ ) is u.d. with respect to $\mu_{1}$ (resp. $\mu$ ).

Definition. We call $\pi: X \rightarrow Y$ open in $x \in X$ if $\pi(U)$ is a neighbourhood of $\pi(x)$ for any neighbourhood $U$ of $x$.

TheOREM 1. Let $X, Y$ be compact, metrizable spaces, $\pi: X \rightarrow Y$ continuous, surjective, $\mu$ a probability measure on $X, \nu=\pi(\mu)$. The following statements are equivalent:

(i) if $\left(y_{n}\right)$ is $\nu$-u.d. in $Y$, then there exists a $\mu$-u.d. sequence $\left(x_{n}\right)$ in $X$, such that $\pi\left(x_{n}\right)=y_{n}$.

(ii) the set of points $x \in X$, where $\pi$ is not open has $\mu$-measure zero.

Proof. (ii) $\Rightarrow$ (i): We fix metrics on $X$ and $Y$ and denote them by the same letter $d$. Let $K_{\varepsilon}(x)$ be the open ball with center $x$ and radius $\varepsilon$. If $\pi$ is not open in $x \in X$, there exists $\varepsilon>0$ such that $\pi(x) \notin \pi\left(K_{\varepsilon}(x)\right)^{\circ}\left({ }^{\circ}\right.$ denotes the interior). Put $M_{\varepsilon}=\left\{x \in X: x \notin \pi^{-1}\left(\pi\left(K_{\varepsilon}(x)\right)^{\circ}\right)\right\}$. By (ii) $\mu\left(M_{\varepsilon}\right)=0$ for all $\varepsilon>0$.

We claim that $M_{\varepsilon}^{-} \subseteq M_{\varepsilon / 2}:$ Assume that $u_{n} \in M_{\varepsilon}$ converges to $u$. For each $u_{n}$ there exists a sequence $v_{n m}$ in $Y$, which converges to $\pi\left(u_{n}\right)$ and does not belong to $\pi\left(K_{\varepsilon}\left(u_{n}\right)\right)$. Selecting an appropriate diagonal sequence, we find elements $w_{n}$, which converge to $\pi(u)$ and such that $w_{n} \notin \pi\left(K_{e}\left(u_{n}\right)\right)$. If $w_{n}=$ $\pi\left(w_{n}^{\prime}\right)$ with $w_{n}^{\prime} \in K_{e / 2}(u)$, then $w_{n}^{\prime} \notin K_{e}\left(u_{n}\right)$ and consequently

$$
d\left(u, u_{n}\right) \geqslant d\left(w_{n}^{\prime}, u_{n}\right)-d\left(u, w_{n}^{\prime}\right) \geqslant \varepsilon / 2 .
$$

This means that $w_{n} \notin \pi\left(K_{\varepsilon / 2}(u)\right)$ for large $n$.

Since $M_{\varepsilon}^{-}$is compact and satisfies $\mu\left(M_{\varepsilon}^{-}\right)=0$, there exists an open set $U_{\varepsilon} \supseteq M_{\varepsilon}^{-}$with $\mu\left(\partial U_{\varepsilon}\right)=0$ and $\mu\left(U_{\varepsilon}\right)<\varepsilon$. There exists a number $\delta(\varepsilon)>0$ 
such that $K_{\delta}(\pi(x)) \subseteq \pi\left(K_{2 \varepsilon}(x)\right)$ for all $x \in U_{\varepsilon}^{\prime}$ : otherwise we can find a sequence $\left(u_{n}\right) \subseteq U_{\varepsilon}^{\prime}$, which converges to some element $u$ and for which $K_{1 / n}\left(\pi\left(u_{n}\right)\right) \underline{Z} \pi\left(K_{2 \varepsilon}\left(u_{n}\right)\right)$. This means that we can choose a sequence $\left(v_{n}\right)$ in $Y$, which converges to $\pi(u)$ and satisfies $v_{n} \notin \pi\left(K_{2 \varepsilon}\left(u_{n}\right)\right)$. As above it follows that $v_{n} \notin \pi\left(K_{\varepsilon}(u)\right)$ for large $n$, contradicting our construction of $U_{\varepsilon}$.

Now assume that $U_{\varepsilon}$ and $\delta(\varepsilon)$ have been chosen for $\varepsilon=1 / n(n=$ $1,2, \ldots)$. If $\left(y_{n}\right)$ is $\nu$-u.d. in $Y$, there exists by Proposition 2 a $\mu$-u.d. sequence $\left(z_{n}\right)$ in $X$, such that $d\left(\pi\left(z_{n}\right), y_{n}\right)$ converges to zero. Since $\mu\left(\partial U_{\varepsilon}\right)=0$, we have

$$
\lim _{N \rightarrow \infty} N^{-1} \sum_{n=1}^{N} c_{U_{\varepsilon}}\left(z_{n}\right)=\mu\left(U_{e}\right)<\varepsilon
$$

for all $\varepsilon>0$. We choose a sequence of indices $N_{k}$ with the following properties:

(1) $d\left(\pi\left(z_{n}\right), y_{n}\right)<\delta(1 / k)$ for $n \geqslant N_{k}$;

(2) $\left(N-N_{k}\right)^{-1} \sum_{n=N_{k}+1}^{N} c_{U_{1 / k}}\left(z_{n}\right)<1 / k$ for $N \geqslant(1+1 / k) N_{k}$;

(3) $N_{k+1} \geqslant(1+1 / k) N_{k}$.

If $N_{k}<n<N_{k+1}$ and $z_{n} \in U_{1 / k}^{\prime}$, we can find $x_{n} \in X$ with $d\left(x_{n}, z_{n}\right)<2 / k$, and $\pi\left(x_{n}\right)=y_{n}$. Otherwise choose an arbitrary $x_{n} \in X$ with $\pi\left(x_{n}\right)=y_{n}$. By (2) the set $\cup_{k=1}^{\infty}\left\{n: N_{k}<n<N_{k+1}, z_{n} \in U_{1 / k}\right\}$ has density zero in N. On the complement of this set the distance from $x_{n}$ to $z_{n}$ tends to zero. It follows that $\left(x_{n}\right)$ is $\mu$-u.d.

(i) $\Rightarrow$ (ii): Assume that $\cup_{\varepsilon>0} M_{\varepsilon}$, i.e. the set of points where $\pi$ is not open has positive outer measure. Then there exists $\varepsilon>0$ such that $M_{2 \varepsilon}$ has positive outer measure. If $x \in M_{2 \varepsilon}$ and $d(x, y)<\varepsilon$, then $K_{\varepsilon}(y) \subseteq K_{2 \varepsilon}(x)$. Consequently $\pi^{-1}\left(\pi\left(K_{\varepsilon}(y)\right)^{\circ}\right) \subseteq \pi^{-1}\left(\pi\left(K_{2 \varepsilon}(x)\right)^{\circ}\right)$ and furthermore $x \in K_{\varepsilon}(y)$ $\backslash \pi^{-1}\left(\pi\left(K_{\varepsilon}(y)\right)^{\circ}\right)$. It follows that $M_{2 \varepsilon} \subseteq \bigcup\left\{K_{\varepsilon}(y) \backslash \pi^{-1}\left(\pi\left(K_{\varepsilon}(y)\right)^{\circ}\right)\right\}$ the union being taken over a countable dense subset of $X$. This shows the existence of an element $y \in X$, for which $\mu\left(K_{\varepsilon}(y) \backslash \pi^{-1}\left(\pi\left(K_{\varepsilon}(y)\right)^{\circ}\right)\right)>0$ for uncountably many $\varepsilon>0$, i.e. we may in addition assume that $\mu\left(\partial K_{\varepsilon}(y)\right)=0$. In this way we have found an open subset $U$ of $X$, with $\mu(\partial U)=0$ and $\mu\left(U \backslash \pi^{-1}\left(\pi(U)^{\circ}\right)\right)>0$.

Now let $\nu_{1}$ be the restriction of $\nu$ to $Y \backslash \pi(U)^{\circ}$ and $\nu_{2}=\nu-\nu_{1}$. Since $Y$ is metrizable, we can find a $\nu$-u.d. sequence $\left(y_{n}\right)_{n \in N}$, which is composed of a $\nu_{1}$-u.d. subsequence $\left(y_{n}\right)_{n \in N_{1}}$ and a $\nu_{2}$-u.d. sequence $\left(y_{n}\right)_{n \in N_{2}}$ such that $y_{n} \in Y \backslash \pi(U)$ for $n \in \mathbf{N}_{1}$ and $y_{n} \notin \partial \pi(U)$ for $n \in \mathbf{N}_{2}$.

Now assume that there exists a $\mu$-u.d. sequence $\left(x_{n}\right)$ in $X$, for which $\pi\left(x_{n}\right)=y_{n}$. By the regularity of $\nu$, there exists an open subset $V$ of $\pi(U)$ such that $\nu(\partial V)=0$ and

$$
\nu\left(\pi(U)^{\circ} \backslash V\right)<\mu\left(U \backslash \pi^{-1}\left(\pi(U)^{\circ}\right)\right) .
$$

Since $\partial\left(\pi^{-1}(V)\right) \subseteq \pi^{-1}(\partial V)$ we have $\mu\left(\partial\left(\pi^{-1}(V)\right)\right)=0$. $\mu(\partial U)=0$, consequently $\lim _{N \rightarrow \infty} N^{-1} \sum_{n=1}^{N} c_{U}\left(x_{n}\right)=\mu(U)$. Now we 
decompose the left side of this equation, using that

$$
\begin{aligned}
U & =\left(U \cap \pi^{-1}(V)\right) \cup\left(U \backslash \pi^{-1}(V)\right) \\
& \subseteq\left(U \cap \pi^{-1}(V)\right) \cup \pi^{-1}(\pi(U) \backslash V) .
\end{aligned}
$$

If $x_{n} \in \pi^{-1}(\pi(U) \backslash V)$, then $y_{n} \in \pi(U)$. By our construction of $y_{n}$ it follows that $y_{n} \in \pi(U)^{\circ}$ and $n \in N_{2}$. Since $\nu_{2}\left(\partial\left(\pi(U)^{\circ}\right)\right)=0$ and clearly $\nu_{2}(\partial V)=0$ the limit corresponding to $U \backslash \pi^{-1}(V)$ can be estimated by $\nu_{2}\left(\pi(U)^{\circ} \backslash V\right)$ and we would get:

$$
\begin{aligned}
\mu(U) & <\mu\left(U \cap \pi^{-1}(V)\right)+\nu_{2}\left(\pi(U)^{\circ} \backslash V\right) \\
& <\mu\left(U \cap \pi^{-1}(V)\right)+\mu\left(U \backslash \pi^{-1}\left(\pi(U)^{\circ}\right)\right) \\
& <\mu(U) \text { a contradiction. }
\end{aligned}
$$

Definition [2]. A topological space $X$ is called dyadic, if there exists some index set $I$ and a continuous surjective map $\pi:\{0,1\}^{I} \rightarrow X$.

Theorem 2. Let $X$ be a dyadic space with a base of cardinality $\aleph_{1}, \mu a$ probability measure on $X$. Then there exists a $\mu$-u.d. sequence $\left(x_{n}\right)$ in $X$.

Proof. By [2], there exists a set $I$ of cardinality $\aleph_{1}$ and a continuous surjective map $\pi:\{0,1\}^{I} \rightarrow X$. By the Hahn-Banach theorem there exists a probability measure $\nu$ on $\{0,1\}^{I}$ such that $\pi(\nu)=\mu$. If $\left(y_{n}\right)$ is $\nu$-u.d. in $\{0,1\}^{I}$, then $\left(\pi\left(y_{n}\right)\right)$ is $\mu$-u.d. in $X$. In this way we have reduced the problem to the case $X=\{0,1\}^{I}$. We identify $I$ with the first uncountable ordinal $\omega_{1}$. For $\alpha<\omega_{1}$ put $X_{\alpha}=\{0,1\}^{\alpha}$. We consider the projections $p_{\alpha}: X \rightarrow X_{\alpha}$ and $p_{\alpha \beta}: X_{\alpha} \rightarrow X_{\beta}(\beta<\alpha)$ and the measures $\nu_{\alpha}=p_{\alpha}(\nu)$. By induction we define $\nu_{\alpha}$-u.d. sequences $\left(x_{n \alpha}\right)$ in $X_{\alpha}$ such that $p_{\alpha \beta}\left(x_{n \alpha}\right)=x_{n \beta}$ : if $\alpha=\beta+1$ for some $\beta$, then we may apply Theorem 1 since $X$ is metrizable and $p_{\alpha \beta}$ open; if $\alpha$ is a limit ordinal, $X_{\alpha}$ is the projective limit of the spaces $X_{\beta}(\beta<\alpha)$ and $\left(x_{n \beta}\right)_{\beta<\alpha}$ defines a unique element $x_{n \alpha}$ in $X_{\alpha}$. By the Stone-Weierstrass theorem $\left(x_{n \alpha}\right)$ is $\nu_{\alpha}$-u.d. In the same way one gets from the sequences $\left(x_{n \alpha}\right)_{\alpha<\omega_{1}}$ a $\nu$-u.d. sequence $\left(x_{n}\right)$. (Cf. the last remark in [10].)

COROLlary. Let $G$ be a compact separable topological group, $\mu$ a probability measure. If we assume the continuum hypothesis, there exists a $\mu$-u.d. sequence in $G$.

Proof. By [8] any compact topological group is a dyadic space, so the result follows from our theorem. One can also give a direct proof if one considers a well-ordered sequence of closed normal subgroups $\left(H_{\alpha}\right)_{\alpha<\omega_{1}}$, such that $G / H_{\alpha}$ is metrizable and $\cap H_{\alpha}=\{e\}$ (i.e. $G$ is the projective limit of the groups $G / H_{\alpha}$ ). (Cf. the proof of Lemma 2 in [9].)

EXAMPLE. If one assumes the continuum hypothesis, there exists a separable compact space $X$ which is not dyadic and such that any probability measure on $X$ admits a u.d. sequence. 
Let $A$ be the algebra of almost periodic functions on $\mathbf{Z}$ and $c_{0}$ the algebra of complex sequences $\left(a_{n}\right)_{n=-\infty}^{\infty}$ for which

$$
\lim _{n \rightarrow \infty} a_{n}=\lim _{n \rightarrow-\infty} a_{n}=0 .
$$

We consider the subalgebra $B$ of $l_{\infty}(Z)$ generated by $A$ and $c_{0} . B$ determines a compactification $X$ of $\mathbf{Z}$. Any maximal ideal in $B$ has either the form $I+c_{0}$, where $I$ is a maximal ideal in $A$ or $A+J$, where $J$ is a maximal ideal in $c_{0}$. It follows that $X$ can be written as a disjoint union of $G$ and $\mathbf{Z}$, where $G$ denotes the Bohr compactification of $\mathbf{Z}$. $\mathbf{Z}$ is open in $X$ and $X$ induces the group topology on $G$. If $\mu$ is a probability measure on $X$, it can be decomposed into measures on $G$ and $\mathbf{Z}$ and by Theorem 2 u.d. sequences exist for both of them. But $X$ is not dyadic, since it is a nonmetrizable compactification of $\mathbf{Z}$ $[3$, p. 61].

Some counterexamples. (1) There exists a probability measure $\mu$ on $X=$ $[0,1]^{c}$ which admits a u.d. sequence (under assumption of the continuum hypothesis), but for which no u.d. sequence exists, which is contained in the support of $\mu$ :

Let $\beta \mathbf{N}$ be the Stone-Čech compactification of the natural numbers and $\mu$ a probability measure on $\beta \mathbf{N}$, which is not concentrated on a countable subset. There exists an injective, continuous map $j: \beta \mathbf{N} \rightarrow X$. By [9, Proposition], there exists no $\mu$-u.d. sequence in $\beta \mathbf{N}$, but by Theorem $2 \mu$ admits a u.d. sequence in $X$.

(2) Let $G$ be the Bohr compactification of the integers. There exist probability measures on $G$, for which no u.d. sequences exist, which are contained in the dense subgroup $\mathbf{Z}$ :

Let $\mu$ be a point measure, concentrated in a point $x \in G \backslash Z$. Assume that $\left(x_{n}\right) \subseteq \mathbf{Z}$ is $\mu$-u.d. The dual group of $G$ is the torus group $T$ (with discrete topology). If $\chi$ is a continuous character on $G$, then $\lim N^{-1} \Sigma_{1}^{N} \chi\left(x_{n}\right)=\chi(x)$. By duality $x_{n}$ and $x$ can be identified with characters on $T$. It follows that $N^{-1} \Sigma_{1}^{N} x_{n}$ converges to $x$, pointwise on $T$. Since $x_{n} \in \mathbf{Z}$, they define continuous characters on $T$ and so $x$ would be Borel-measurable. But by [5, Corollary 22.19, p. 346] a Borel measurable character is already continuous, which contradicts $x \notin \mathbf{Z}$.

(3) There exists a dense subset $M$ in $\{+1,-1\}^{c}$, such that there exists no sequence in $M$, which is u.d. with respect to the product measure:

If $\left(x_{n}\right)$ is a dense sequence in $\{+1,-1\}^{c}$, it defines a system $\left(A_{\alpha}\right)_{\alpha \in c}$ of subsets of $\mathbf{N}$ by $A_{\alpha}=\left\{n: x_{n \alpha}=1\right\}$ (where $x_{n}=\left(x_{n \alpha}\right)_{\alpha \in c}$ ). If $A$ is a subset of $\mathbf{N}$, we use the notation $A^{1}=A, A^{-1}=\mathbf{N} \backslash A$. Now if $\alpha_{1}, \ldots, \alpha_{k}$ are different indices in $c$, and $i_{1}, \ldots, i_{k} \in\{+1,-1\}$, then $A_{\alpha_{1}}^{i_{1}} \cap \cdots \cap A_{\alpha_{k}}^{i_{k}}=$ $\left\{n: x_{n \alpha_{1}}=i_{1}, \ldots, x_{n \alpha_{k}}=i_{k}\right\} \neq \varnothing$.

Conversely let $\mathfrak{A}$ be a family of subsets of $\mathbf{N}$, with cardinality $c$ and which is maximal with respect to the property that $A_{\alpha_{1}}^{i_{1}} \cap \cdots \cap A_{\alpha_{k}}^{i_{k}} \neq \varnothing$ for any 
distinct elements $A_{\alpha_{1}}, \ldots, A_{\alpha_{k}} \in \mathfrak{U}$ and $i_{1}, \ldots, i_{k} \in\{+1,-1\}$. (We index the elements of $\mathfrak{A}$ by the elements of $c)$. $\mathfrak{A}$ defines a sequence $\left(x_{n}\right)$ in $\{+1,-1\}^{c}$ by $x_{n \alpha}=1$ if $n \in A_{\alpha}$ and $x_{n \alpha}=-1$ if $n \notin A$. If $\alpha_{1}, \ldots, \alpha_{k} \in c$ and $i_{1}, \ldots, i_{k} \in\{+1,-1\}$ then $\left\{n: x_{n \alpha_{1}}=i_{1}, \ldots, x_{n \alpha_{k}}=i_{k}\right\}=A_{\alpha_{1}}^{i_{1}}$ $\cap \cdots \cap A_{\alpha_{k}}^{i_{k}} \neq \varnothing$. It follows that $M=\left\{x_{n}\right\}$ is dense in $\{+1,-1\}^{c}$.

Assume that $\left(y_{n}\right)$ is a u.d. sequence in $\{+1,-1\}^{c}$, which is contained in $M$, i.e. we have a map $p: \mathbf{N} \rightarrow \mathbf{N}$ such that $y_{n}=x_{p(n)}$. Put $\beta_{i n}=n^{-1} \mid\{k<n$ : $p(k)=i\} \mid$. Then $\sum_{i=1}^{\infty} \beta_{i n}=1$ for all $n$ and if $n$ is fixed, $\beta_{i n} \neq 0$ holds only for a finite number of $i \in \mathbf{N}$. We have

$$
\lim _{n \rightarrow \infty} \sum_{i=1}^{\infty} \beta_{i n} f\left(x_{i}\right)=\lim _{n \rightarrow \infty} n^{-1} \sum_{k=1}^{n} f\left(y_{k}\right)=\int f d \mu
$$

for all continuous or Riemann integrable functions $f:\{+1,-1\}^{c} \rightarrow \mathbf{R}$. If one takes the characteristic function of $x_{i}$, it follows that $\lim _{n \rightarrow \infty} \beta_{i n}=0$.

Now we claim that there exists a subset $B$ of $\mathbf{N}$ for which

$$
\underset{n \rightarrow \infty}{\limsup } \sum_{i=1}^{\infty} \beta_{i n} c_{B}(i)=1 \text { and } \liminf _{n \rightarrow \infty} \sum_{i=1}^{\infty} \beta_{i n} c_{B}(i)=0 \text {. }
$$

The set $B$ is constructed by induction: Assume that a subset $B_{n}$ of the interval $[1, N]$, and $\varepsilon>0$ are given. Since $\lim _{m \rightarrow \infty} \beta_{i m}=0$, there exists $m>n$, such that $\beta_{i m}<\varepsilon N^{-1}$ for all $i<N$. Put $M=\max \left\{i: \beta_{i m}>0\right\} \cup\{N\}$. If we write $B_{m}$ for $B_{n}$, regarded as a subset of $[1, M]$, then $\sum_{i=1}^{\infty} \beta_{i m} c_{B_{m}}(i)<\varepsilon$. On the other hand, if we put $B_{m}=B_{n} \cup[N+1, M]$, then

$$
\sum_{i=1}^{\infty} \beta_{i m} c_{B_{m}}(i) \geqslant \sum_{i=N+1} \beta_{i m}=1-\sum_{i=1}^{N} \beta_{i m} \geqslant 1-\varepsilon .
$$

Both sums do not change their value if we add a subset of $\left[M+1, \infty\left[\right.\right.$ to $B_{m}$. If one applies alternately these two enlargement procedures and $\varepsilon$ tends to zero, one gets the desired set $B$.

If $B=A_{\alpha} \in \mathfrak{A}$, then $\lim _{m \rightarrow \infty} \sum_{i=1}^{\infty} \beta_{i m} c_{B}(i)=\lim _{m \rightarrow \infty} m^{-1}\left|\left\{k: y_{k \alpha}=1\right\}\right|$ $=2^{-1}$ which is impossible. Since $\mathfrak{A}$ is maximal, there exist $\alpha_{1}, \ldots, \alpha_{k} \in c$, $i_{0}, i_{1}, \ldots, i_{k} \in\{+1,-1\}$ such that $B^{i_{0}} \cap A_{\alpha_{1}}^{i_{1}} \cap \cdots \cap A_{\alpha_{k}}^{i_{k}}=\varnothing$. Replacing $B$ by $\mathbf{N} \backslash B$ if necessary, we may assume that $i_{0}=-1$ i.e. $B \subseteq A_{\alpha_{k}}^{i_{1}} \cap \cdots \cap$ $A_{\alpha_{k}}^{i_{k}}$. But then

$$
\limsup _{m \rightarrow \infty} \sum_{i=1}^{\infty} \beta_{i m} c_{B}(i)<\lim _{m \rightarrow \infty} m^{-1} \mid\left\{j: y_{j \alpha_{1}}=i_{1}, \ldots, y_{j \alpha_{k}}=i_{k}\right\}=2^{-k}
$$

which is again a contradiction.

\section{REFERENCES}

1. L. Benzinger, Uniformly distributed sequences in locally compact groups. I, Trans. Amer. Math. Soc. 188 (1974), 149-165.

2. B. A. Efimov and R. Engelking, Remarks on dyadic spaces. II, Colloq. Math. 13 (1964/65), 181-197. 
3. R. Engelking and A. PeYczyński, Remarks on dyadic spaces, Colloq. Math. 11 (1963), 55-63.

4. P. Gerl, Relative Gleichverteilung in lokalkompakten Räumen, Math. Z. 121 (1971), 24-50.

5. E. Hewitt and K. A. Ross, Abstract harmonic analysis. Vol. I, Academic Press, New York; Springer, Berlin, 1963.

6. E. Hlawka, Zur formalen Theorie der Gleichverteilung in kompakten Gruppen, Rend. Circ. Mat. Palermo (2) 4 (1955),33-47.

7. L. Kuipers and H. Niederreiter, Uniform distribution of sequences, Wiley, New York, 1974.

8. V. Kuzminov, Aleksandrov's hypothesis in the theory of topological groups, Dokl. Akad. Nauk SSSR 125 (1959), 727-729. (Russian)

9. V. Losert, Uniformly distributed sequences on compact, separable, non metrizable groups, Acta Sci. Math. (Szeged) 40 (1978), 107-1 10.

10. V. Losert and H. Rindler, Teilfolgen gleichverteilter Folgen, J. Reine Angew. Math. (to appear).

11. H. Rindler, Uniform distribution on locally compact groups, Proc. Amer. Math. Soc. 57 (1976), 130-132.

12. W. A. Veech, Some questions of uniform distribution, Ann. of Math. (2) 94 (1971), 125-138.

Mathematisches Institut der Universitït Wien, A-1090 Wien, Strudlhofgasse 4, AUSTRIA 